\title{
Dynamics of an Unbounded Interface Between Ordered Phases
}

\author{
P. L. Krapivsky $\mathbb{A}^{7}$ and S. Rednel] \\ Center for BioDynamics, Center for Polymer Studies, \\ and Department of Physics, Boston University, Boston, MA 02215, USA \\ J. Tailleur \\ Departement de Physique, ENS de Cachan, 61 avenue du President Wilson, 94235 Cachan Cedex, Paris, France
}

\begin{abstract}
We investigate the evolution of a single unbounded interface between ordered phases in twodimensional Ising ferromagnets that are endowed with single-spin-flip zero-temperature Glauber dynamics. We examine specifically the cases where the interface initially has either one or two corners. In both examples, the interface evolves to a limiting self-similar form. We apply the continuum time-dependent Ginzburg-Landau equation and a microscopic approach to calculate the interface shape. For the single corner system, we also discuss a correspondence between the interface and the Young diagram that represents the partition of the integers.
\end{abstract}

PACS numbers: 64.60.My, 05.50.+q, 75.40.Gb

\section{INTRODUCTION}

At low temperatures, interfaces between two brokensymmetry ordered phases typically shrink and eventually disappear [1]. The dynamics is usually driven by forces that reduce the interface and leads to surprisingly complicated coarsening processes - even the evolution of an isolated simply-connected domain of minority phase in a sea of the majority phase is in general insoluble. However, every finite domain of linear size $R$ disappears in a finite time that scales as $R^{2}$ [2] for dynamics that does not conserve the order parameter [3]. In this sense, we understand the shrinking of a single domain, or equivalently, the evolution of a single bounded interface [4] .

The goal of this work is to understand the evolution of a single unbounded two-dimensional interface in simple geometric configurations. The most elementary such example is an infinite straight interface. For this geometry, any spin flip event increases the length of the interface and raises the energy. Thus a straight interface does not evolve - to have any evolution at zero temperature, the interface must have curvature. The simplest realization of a curvature in a lattice system is an infinite interface with a single corner (Fig. 11). While not a direct analog of the theoretical models we consider in this paper, a physical realization of such a geometry is the spreading of a fluid in a V-shaped groove [5].

According to zero-temperature single-spin-flip Glauber dynamics [3], the corner spin $\sigma_{0,0}$ can flip. After it flips, the neighbors $\sigma_{0,1}$ and $\sigma_{1,0}$ can flip, or $\sigma_{0,0}$ can flip back, etc. The interface thus evolves stochastically and can, in principle, return to its original configuration as time increases $[\underline{6}$. This is, however, exceedingly improbable and generically the interface recedes diffu-

\footnotetext{
*Electronic address: paulk@bu.edu

${ }^{\dagger}$ Electronic address: redner@bu.edu

${ }^{\ddagger}$ Electronic address: Julien.Tailleur@crans.org
}
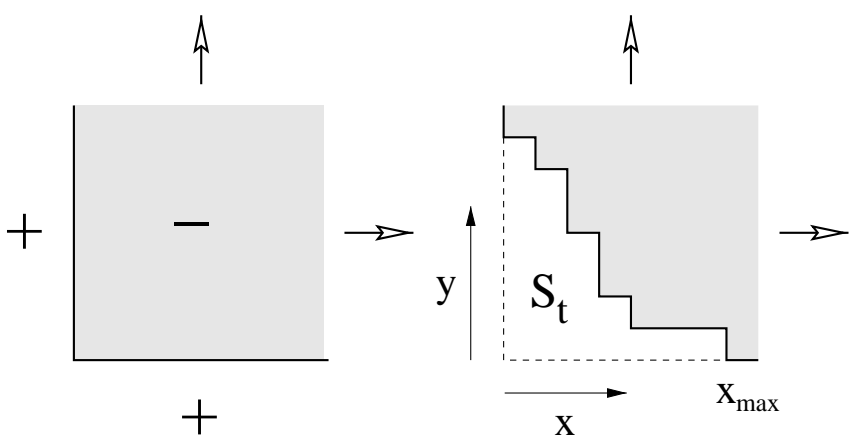

FIG. 1: A single corner interface (left) in the initial state and some time later (right). Grey denotes spin down (extending to $\infty$ in the $+x$ and $+y$ directions), and white denotes spin up. The evolving interface encloses an area $S_{t}$ at time $t$.

sively (since the dynamics does not conserve the order parameter), i.e., $x \propto \sqrt{t}$ and $y \propto \sqrt{t}$. Furthermore, although the interface at a fixed time fluctuates from realization to realization, it becomes progressively less random as time increases. More precisely, after the contraction $(x, y) \rightarrow(x / \sqrt{t}, y / \sqrt{t})$ the interface approaches a deterministic limiting shape. In the following two sections, we will study the time evolution of the interface and determine its shape within a continuum (Sec. II) and a microscopic approach (Sec. III). In addition to the wedge geometry, we will study, in Sec. IV, interface evolution in systems that initially contain two corners the macroscopic step and the semi-infinite finger geometries. Finally, in Sec. V, we study the time dependence of fluctuations in the interface shape.

\section{COARSE-GRAINED DESCRIPTION}

A natural way to study interface evolution is through the time-dependent Ginzburg-Landau (TDGL) equation [1]. It is generally believed that the long-time behav- 
ior predicted by the continuum TDGL equation should be the same as that for the microscopic Ising-Glauber model. In the case of the single interface, we find, surprisingly, that the predictions of the TDGL description qualitatively disagree with simulations of the Ising interface that evolves by zero-temperature Glauber kinetics.

Since we are primarily interested in interfacial behavior, we will not write the TDGL equation but instead will merely utilize a reduction directly to the interface dynamics. As found by Allen and Cahn [7], the normal velocity of the interface is proportional to the local curvature, that is,

$$
v_{n}=-D \nabla \cdot \mathbf{n},
$$

where $D$ is the diffusion constant and $\mathbf{n}$ is the local normal to the interface [8]. This interface dynamics in the TDGL equation is a specific example of curvature-driven flow [9, 10, 11, 12, 13], where one is concerned with the evolution of general shapes in arbitrary dimension due to a local velocity that is proportional to the local curvature.

For the case of interest to us, namely, a onedimensional interface whose locus is $y(x, t)$, the curvature is

$$
\nabla \cdot \mathbf{n}=-\frac{y_{x x}}{\left[1+y_{x}^{2}\right]^{3 / 2}},
$$

where the subscripts denote partial differentiation. Using the kinematic condition $v_{n} \sqrt{1+y_{x}^{2}}=y_{t}$ we find that the interface $y(x, t)$ obeys the diffusion-like equation

$$
y_{t}=D \frac{y_{x x}}{1+y_{x}^{2}} .
$$

Because of the absence of any constant with dimension of length in this equation, the corresponding solution admits the self-similar form

$$
y(x, t)=\sqrt{D t} Y(X), \quad X=x / \sqrt{D t} .
$$

Note that the increase of the magnetization is equal to twice the area under the curve $y(x, t)$. From ansatz (4), the growth of the area is proportional to $t$, so that the magnetization also grows linearly with time.

To solve the equation of motion (3), we substitute into this equation the ansatz of Eq. (44) and find that the scaling function $Y(X)$ obeys

$$
\frac{Y-X Y^{\prime}}{2}=\frac{Y^{\prime \prime}}{1+\left(Y^{\prime}\right)^{2}}
$$

where prime indicates differentiation with respect to $X$. Equation (5) should be solved subject to the constraints

$$
\lim _{X \rightarrow \infty} Y(X)=0, \quad \lim _{X \rightarrow+0} Y(X)=\infty .
$$

Thus we recast the original problem in Eq. (3) into an ordinary differential equation subject to the above boundary conditions. Within the TDGL equation framework we note that one can also study the evolution of a wedge with an arbitrary opening angle. For example, if the wedge initially occupies the region $y>|x| \tan \theta$ we should solve Eq. (5) subject to the boundary condition $Y \rightarrow \pm X \tan \theta$ as $X \rightarrow \pm \infty$.

To solve Eq. (5), we first introduce the polar coordinates $(X, Y)=(r \cos \theta, r \sin \theta)$ and after straightforward variable transformations we recast Eq. (5) into the following equation for $r=r(\theta)$ :

$$
2 r \frac{d^{2} r}{d \theta^{2}}-\left(4+r^{2}\right)\left(\frac{d r}{d \theta}\right)^{2}=r^{2}\left(2+r^{2}\right) .
$$

Writing $\frac{d r}{d \theta}=R(r)$, further reduces Eq. (7) to the firstorder equation

$$
\left(r \frac{d}{d r}-r^{2}-4\right) R^{2}=r^{2}\left(2+r^{2}\right)
$$

whose solution is

$$
R^{2}=r^{4} e^{r^{2} / 2} F\left(r, r_{*}\right),
$$

with

$$
F\left(r, r_{*}\right)=\int_{r_{*}}^{r} d \rho\left(\frac{2}{\rho^{3}}+\frac{1}{\rho}\right) e^{-\rho^{2} / 2} .
$$

The interface is now determined from

$$
\frac{d r}{d \theta}=-r^{2} e^{r^{2} / 4} \sqrt{F\left(r, r_{*}\right)}
$$

for $\theta \leq \pi / 4$. For $\theta \geq \pi / 4$, there should be a plus sign on the right-hand side. Integrating Eq. (11) we arrive at the explicit equation for $\theta=\theta(r)$

$$
\theta=\int_{r}^{\infty} d \rho \rho^{-2} e^{-\rho^{2} / 4}\left[F\left(\rho, r_{*}\right)\right]^{-1 / 2}
$$

for $\theta \leq \pi / 4$. For $\pi / 4<\theta<\pi / 2$, the interface is symmetric with respect to the diagonal, that is, $r(\theta)=r\left(\frac{\pi}{2}-\theta\right)$. The solution in Eq. (12) contains the unknown $r_{*}$, which is the scaled distance from the origin to the closest point on the interface. Its value is obtained by ensuring that $\theta=\pi / 4$ when $r=r_{*}$. This gives the criterion

$$
\int_{r_{*}}^{\infty} d r r^{-2} e^{-r^{2} / 4}\left[F\left(r, r_{*}\right)\right]^{-1 / 2}=\frac{\pi}{4},
$$

whose numerical solution is $r_{*} \approx 1.0445$. Equation (12), with $F$ given by (10), provides an explicit representation of $\theta(r)$ on the interface in terms of the (scaled) distance $r \in\left[r_{*}, \infty\right)$ from the origin.

In the asymptotic regime $r \rightarrow \infty$, the form of the interface becomes much simpler. From Eqs. (10) and (12) we find

$$
\theta \rightarrow A r^{-3} e^{-r^{2} / 4}
$$


with $A=2\left[F\left(\infty, r_{*}\right)\right]^{-1 / 2} \approx 2.74404$. Equivalently,

$$
Y \rightarrow A X^{-2} \exp \left[-\frac{X^{2}}{4}\right] .
$$

Apart from the numerical factor $A$, this behavior can be established directly from Eq. (5D) after dropping the subdominant terms in the asymptotic limit.

While the spatial extent of the continuum interface, defined as the region with non-zero curvature, is strictly infinite, the presence of a lattice cutoff implies that the interface will have a finite extent. The finiteness of the interface may be quantified by the distance of its leading edge, $x_{\max }$ (or $y_{\max }$ ), from the origin (see Fig. 11). We may estimate this distance as the value of $x$ for which the TDGL description first gives $y(x)<a$, where $a$ is the lattice spacing. Substituting the criterion $y=a$ into Eq. (15) we thereby obtain

$$
\frac{a}{\sqrt{D t}}=\frac{A D t}{x_{\max }^{2}} e^{-x_{\max }^{2} / 4 D t}
$$

which leads to the asymptotic behavior

$$
x_{\max } \approx \sqrt{2 D t \ln \left(D t / a^{2}\right)} .
$$

Notice that the value of the lattice spacing is immaterial for the asymptotic behavior and we therefore set $a=1$ henceforth.

\section{MICROSCOPIC DESCRIPTION}

\section{A. Basic Characteristics and Generalizations}

At the microscopic level, the interface has a staircase shape (Fig. 1). Zero-temperature Glauber spin-flip dynamics [3] forbids energy raising flips, so that only spins in the corners on the interface can evolve. While both energy decreasing and energy conserving flips are generically allowed, only energy conserving spin flips can occur in the wedge geometry. We define the rate for these events to be one without loss of generality. The construction of the system ensures that the total number of possible flips of minority spins always exceeds the total number of possible flips of majority spins by one (see Fig. (2). Hence the total number $S_{t}$ of spins in the first quadrant that join the majority phase is a random variable that undergoes a random walk on the half-lattice $S_{t} \in \mathbf{Z}_{+}$, with a constant positive bias that equals one. Thus the expected number of spins that have flipped at time $t$ is $\left\langle S_{t}\right\rangle=t$.

Algorithmically, Glauber kinetics involves randomly picking a spin on the corners of the staircase and allowing this spin to flip freely. Equivalently, we can view the staircase evolution as a deposition/evaporation process in which deposition can occur at sites $x$ where $y(x)<y(x-1)$ (with $y(-1)$ defined to be infinite), while evaporation can occur at sites where $y(x)>y(x+1)$

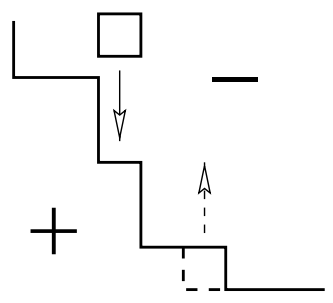

FIG. 2: The elementary steps of deposition and evaporation that drive the evolution of a staircase. In this example, deposition (equivalent to the spin flip event $-\rightarrow+$ ) can occur at 4 sites while evaporation can occur at 3 sites.

(Fig. 2). Here deposition is equivalent to the spin flip process $-\rightarrow+$ and vice versa for evaporation.

According to zero-temperature Glauber kinetics, deposition and evaporation events must occur at the same rate for all eligible sites. Owing to the above-mentioned fact that there is always exactly one more site available for deposition than for evaporation, this "unbiased" evolution rule leads to a steadily growing interface in which the average number of particles in the deposit grows as $t$. This particulate description for the interface naturally suggests the generalization to different deposition and evaporation rates. For reasons that will soon become evident, we consider the following three rules:

(i) Equal deposition and evaporation rates. This is just evolution of the interface by Glauber kinetics at zero temperature and zero magnetic field.

(ii) Evaporation rate greater than deposition rate. With this rate bias, the interface reaches an equilibrium state.

(iii) Irreversible deposition with no evaporation events.

Physically, rule (iii) is equivalent to interface evolution by zero-temperature Glauber kinetics in the presence of a magnetic field that favors the majority spin; the magnitude of the field is irrelevant (at zero temperature) as long as it is smaller than a threshold value to ensure the stability of flat interfaces.

\section{B. Relation to Partitions}

The staircase can also be viewed as a geometric representation of the partition of the integer number $S_{t}$. This partition is simply the set of $S_{t}$ boxes in the first quadrant that are arranged in non-increasing order. Such an object is also called a Young diagram [14]. For example, the interface of Fig. 3 corresponds to the partitioning of the integer 22 into the set $\{7,6,4,2,1,1,1\}$.

Much is known about partitions [15]; for example, the number of partitions $p(N)$ of the integer $N$ has the asymptotic behavior $p(N) \sim N^{-1} \exp (2 \pi \sqrt{N / 6})$. The asymptotic behavior of Young diagrams is also qualitatively simple: After a suitable rescaling, a typical Young 


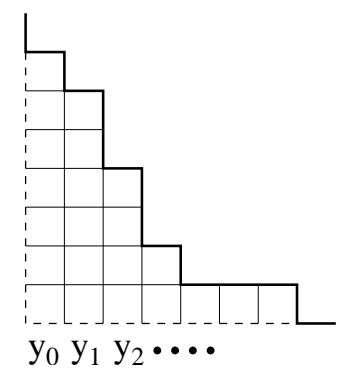

FIG. 3: The Young diagram that is based on the interface profile of Fig. 11 This diagram corresponds to a partition of the integer 22 into the set $\{7,6,4,2,1,1,1\}$.

diagram converges to a limiting shape (see [16] for precise statements and earlier references). To compute this shape one must know the weights of all possible partitions. For the case where each partition occurs with equal weight $1 / p(N)$ (the uniform measure), the corresponding limiting shape is known [16], and will be quoted in the next subsection.

For the Ising interface (rule (i) in the above list), we do not know the weights of each interface configuration [17. We will see that the shape of the Ising interface is slightly different from that of Young diagrams, implying that the weights are not uniform. On the other hand, if the spin flip event $+\rightarrow-$ is favored over $-\rightarrow+$ (rule (ii)), then the interface approaches an equilibrium state. Because the state space is sampled more extensively in the equilibrium system, this suggests that the weights for each interface configuration should be more uniform than in the case of rule (i), as borne out in our simulation results below.

\section{Limiting Shape}

We now present a heuristic derivation for the limiting shape that corresponds to the uniform measure. We follow an argument by Shlosman [18]; see also [19] for a similar approach. The assumption that the measure is uniform implies that we can disregard the underlying dynamics and simply count the number of possible staircases. (A similar calculation for the triangular lattice is presented in the Appendix.)

On the square lattice, the interface is a staircase in $\mathbf{Z}^{2}$ with each step going either to the right or downward (Fig. (1). Let $\mathbf{1}=\left(x_{1}, y_{1}\right)$ and $\mathbf{2}=\left(x_{2}, y_{2}\right)$ be two points in $\mathbf{Z}^{2}$ that can be connected by a staircase; that is, $0 \leq$ $x_{1} \leq x_{2}$ and $y_{1} \geq y_{2} \geq 0$. The number of staircases from $\mathbf{1}$ to $\mathbf{2}$ is

$$
N(\mathbf{1}, \mathbf{2})=\left(\begin{array}{c}
x_{2}-x_{1}+y_{1}-y_{2} \\
x_{2}-x_{1}
\end{array}\right)
$$

For compactness, we set $a=x_{2}-x_{1}$ and $b=y_{1}-y_{2}$. If the points $\mathbf{1}$ and $\mathbf{2}$ are distant, i.e., $a \gg 1$ and $b \gg 1$, the Stirling formula gives

$$
\ln \left(\begin{array}{c}
a+b \\
a
\end{array}\right) \longrightarrow-a \ln \frac{a}{a+b}-b \ln \frac{b}{a+b} .
$$

Therefore

$$
\ln N(\mathbf{1}, \mathbf{2})=\ln \left(\begin{array}{c}
a+b \\
a
\end{array}\right) \longrightarrow \sqrt{a^{2}+b^{2}} \Phi(\mathbf{n}),
$$

where $\mathbf{n}=\left(n_{1}, n_{2}\right)=(b, a) / \sqrt{a^{2}+b^{2}}$ is the unit vector orthogonal to $\mathbf{1}-\mathbf{2}$ and

$$
\Phi(\mathbf{n})=-n_{1} \ln \frac{n_{1}}{n_{1}+n_{2}}-n_{2} \ln \frac{n_{2}}{n_{1}+n_{2}} .
$$

Suppose now that points $\mathbf{1}$ and $\mathbf{2}$ are far enough apart to ensure the applicability of Eq. (18) yet close enough to guarantee that the interface is locally almost flat. Under these conditions, we have

$$
\left(n_{1}, n_{2}\right)=\left(-\frac{y_{x}}{\sqrt{1+y_{x}^{2}}}, \frac{1}{\sqrt{1+y_{x}^{2}}}\right) .
$$

Generally, consider an interface that goes through the points $1, \ldots, \mathbf{k}$, with adjacent points satisfying the above requirements. The total number of these staircases is then the product of the factors $N(\mathbf{j}, \mathbf{j}+\mathbf{1})$. The logarithm of the number of staircases is therefore the sum of these factors (asymptotically an integral). Using Eqs. (18) and (20) we thereby find that the logarithm of the total number of staircases approaches to

$$
\begin{aligned}
G[y] & =\int_{0}^{\infty} d x \sqrt{1+y_{x}^{2}} \Phi\left(-\frac{y_{x}}{\sqrt{1+y_{x}^{2}}}, \frac{1}{\sqrt{1+y_{x}^{2}}}\right) \\
& =\int_{0}^{\infty} d x\left[y_{x} \ln \frac{-y_{x}}{1-y_{x}}-\ln \frac{1}{1-y_{x}}\right] .
\end{aligned}
$$

Since $x, y \propto \sqrt{t}$ on the interface, the number of staircases near the typical interface scales as $e^{\sqrt{t} G[Y]}$. Because $G[Y]$ is of the order of one, the number of staircases rapidly grows with $t$ and the dominant contribution arises from the staircases close to the curve $y=y(x, t)$ that maximizes the functional of Eq. (21). Thus we need to determine only the optimal curve to find the asymptotic interface shape.

In maximizing (21) we must only use curves that bound an area equal to $t$, i.e., $\int_{0}^{\infty} d x y(x, t)=t$. With this isoperimetric constraint, the proper functional to maximize is $G_{\lambda}[y] \equiv G[y]-\lambda \int_{0}^{\infty} d x y$, where $\lambda$ is a Lagrange multiplier. Re-writing $G_{\lambda}[y]=\int_{0}^{\infty} d x L\left(y, y_{x}\right)$, with the Lagrangian

$$
L\left(y, y_{x}\right)=y_{x} \ln \frac{-y_{x}}{1-y_{x}}-\ln \frac{1}{1-y_{x}}-\lambda y,
$$

and applying the Euler-Lagrange formalism, gives the extremum condition $\frac{d}{d x} \ln \frac{-y_{x}}{1-y_{x}}=-\lambda$. Integrating this equation subject to $y_{x}(0)=-\infty$ yields

$$
\frac{-y_{x}}{1-y_{x}}=e^{-\lambda x} \text {. }
$$




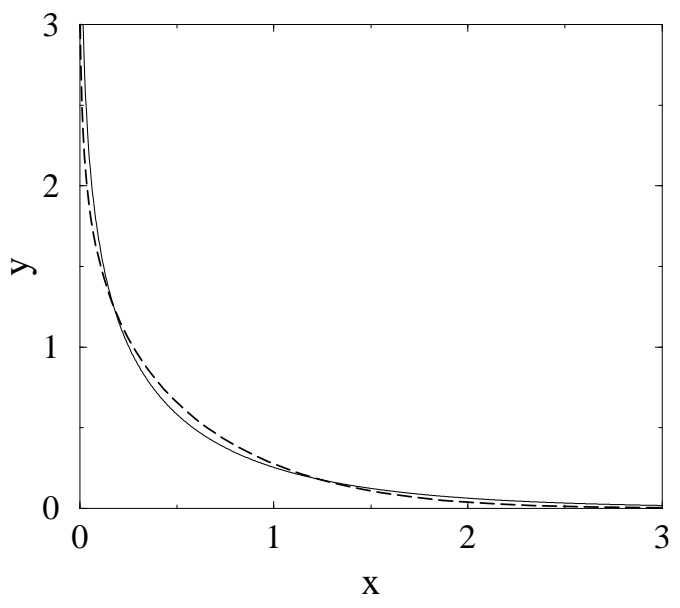

FIG. 4: Comparison of the interfaces from the TDGL equation (Eq. (12) (dashed) and that from Eq. (24) (solid). Both curves are normalized to unit enclosed area.

Integrating (23) subject to $y(\infty)=0$ gives the remarkably simple form [18] for the shape of the optimal staircase (fig. (4)

$$
e^{-\lambda x}+e^{-\lambda y}=1, \quad \text { with } \quad \lambda=\frac{\pi}{\sqrt{6 t}},
$$

where $\lambda$ is determined from the area constraint.

This limiting shape is quantitatively close to that obtained from the coarse-grained TDGL approach, except near the tail region, where the TDGL equation predicts a Gaussian tail (15) while (24) gives an exponential tail

$$
Y \rightarrow \frac{\sqrt{6}}{\pi} \exp \left[-\frac{\pi}{\sqrt{6}} X\right]
$$

as $X \rightarrow \infty$, where $X=x / \sqrt{t}$ and $Y=y / \sqrt{t}$ are the scaled coordinates. Correspondingly, the location of the leading edge is

$$
x_{\max } \rightarrow C \sqrt{t} \ln t
$$

with $C=\sqrt{6} / 2 \pi=0.3898 \ldots$. Notice that the leading edge moves slightly faster than the $\sqrt{t \ln t}$ law predicted by the TDGL approach.

\section{Simulation Results}

We simulated Ising interfaces that are grown by the three different rules defined in Sec. III.A. By rescaling each of these interfaces to have unit area, our numerical results exhibit data collapse after a short-time transient, with each rule giving a slightly different, although quantitatively similar, universal curve. Interestingly, only in the evaporation-dominated case (rule (ii)), does the interface shape coincide with Eq. (24).

It is also worth noting the following subtlety in our measurements of interfaces. In each realization, we
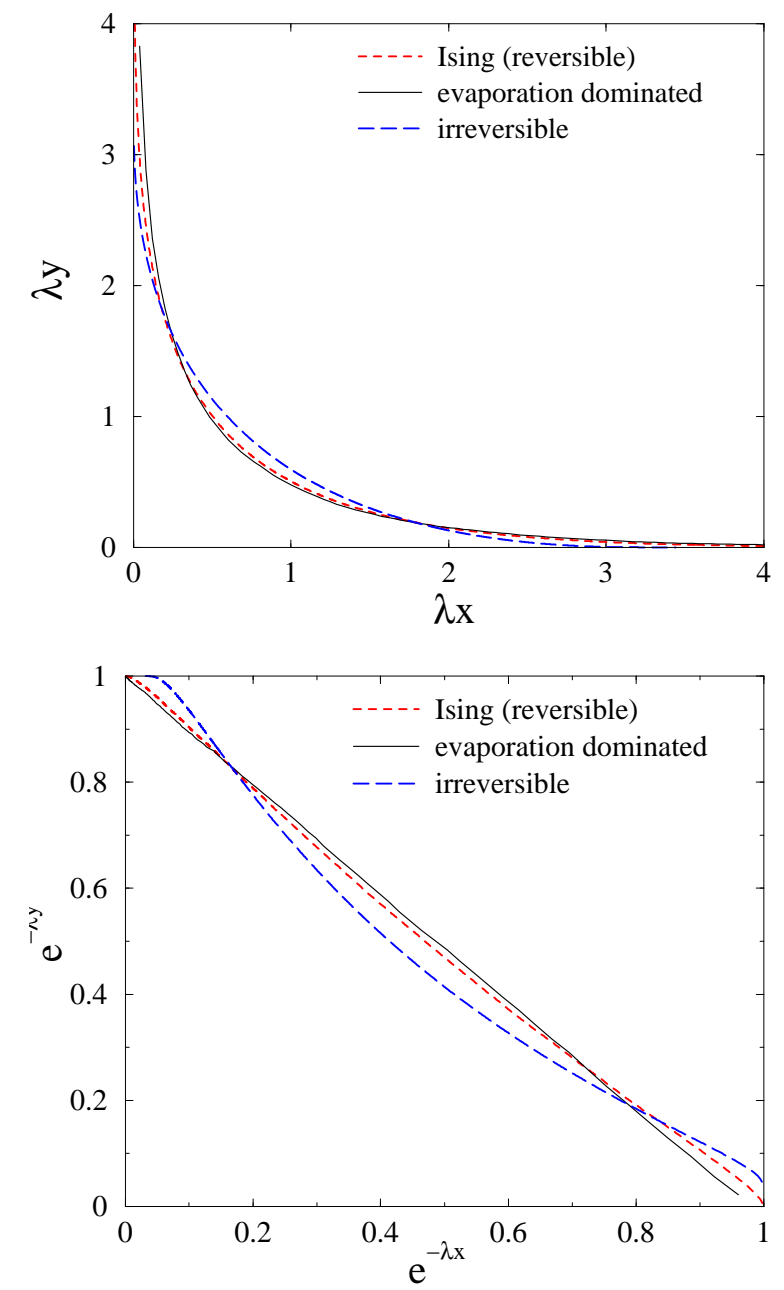

FIG. 5: The interface in the wedge geometry for: (i) equal deposition and evaporation rates - equivalently, the IsingGlauber interface (based on 100 realizations of $4340331 \approx$ $1.5^{32}$ particles), (ii) evaporation rate exceeding the deposition rate (based on 500 realizations and the ratio of evaporation to deposition rate equal to $0.51 / 0.49 \approx 1.041$, corresponding to an average deposit of 1012 particles), and (iii) irreversible deposition (based on $10^{4}$ realizations of $287627=1.5^{31}$ particles). The two plots show the interface on a linear (top) and an exponential scale (bottom). The straight line behavior in the latter corresponds to Eq. (24).

record the height $y(x)$ at each value of $x$, and then we average over many realizations to obtain the average interface $\{\langle y(x)\rangle\}$. This procedure is manifestly not symmetrical about the $45^{\circ}$ diagonal. For example, in our definition of the average profile there is necessarily a non-zero contribution at $x_{\max }$, so that the average profile in the $x$-direction extends to $x_{\max }$. On the other hand, the average profile in the $y$-direction extends only to the smaller value $\langle y(x=1)\rangle$. The asymmetry caused by this averaging is small, except near the extremes of the interface (if one looks closely at Fig. (5). 


\section{RELATED GEOMETRIES}

The next level of complexity is to consider an initial interface with two corners. We specifically study two cases: (a) a single large step that smooths out to an error function profile, and (b) a semi-infinite rectangular "finger" that evolves to a constantly receding steady shape.

\section{A. Single step}

We form a single step interface by two horizontal halflines, $x \leq 0, y=2 h$ and $x \geq 0, y=0$, and the vertical interval $x=0,0 \leq y \leq 2 h$ (Fig. 6). If the height $2 h$ of the step is small, the problem is best analyzed by random walk techniques. For example, when $2 h=2$, the kink in the interface is equivalent to a single particle that undergoes a discrete one-dimensional random walk. Similarly when $2 h=4$ the system consists of two random walks, located say at $x_{L}$ and $x_{R}$, but subject to the constraint that the two particles cannot interchanges their positions.

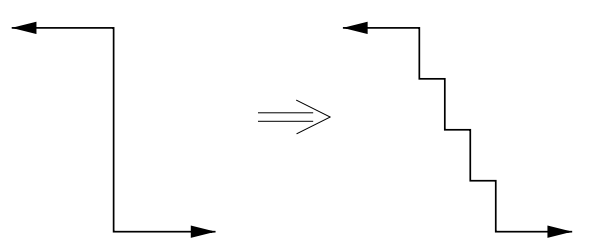

FIG. 6: Schematic evolution of a single step according to Ising-Glauber dynamics.

More interesting behaviors occur for $h \gg 1$, where three regimes arise. When $1 \ll t \ll h^{2}$, the two corners do not 'feel' each other and the problem reduces to that of two non-interacting quadrants. We may obtain a better upper bound for this non-interacting regime by using the growth law for the position of the leading edge (Eq. (16)). Since the unperturbed initial vertical interval starts at $y \approx C \sqrt{t} \ln t$ and ends at $y \approx 2 h-C \sqrt{t} \ln t$, the two corners remain independent as long as $\sqrt{t} \ln t \ll h$, or $t \ll h^{2} / \ln h$.

When $h \sim \sqrt{t}$, the corners interact. In this second regime, we can still determine the interface shape by employing the maximization procedure of section III. To find $y=y(x, t)$ for $x>0$, we should maximize the functional $G_{\lambda}[y]=\int_{0}^{\infty} d x L\left(y, y_{x}\right)$, with the Lagrangian given by (22), but now with the boundary conditions $y(0)=h$ (by symmetry) and $y(\infty)=0$. We find

$$
\left(1-e^{-\lambda h}\right) e^{-\lambda x}+e^{-\lambda y}=1
$$

The area bounded by this curve is

$$
\int_{0}^{\infty} d x y=\lambda^{-2} \operatorname{Li}_{2}\left(1-e^{-\lambda h}\right)
$$

where $\operatorname{Li}_{2}(z)=\sum_{n \geq 1} z^{n} / n^{2}$ is the dilogarithm function. Equating the area to $t$ we obtain

$$
\Lambda^{-2} \operatorname{Li}_{2}\left(1-e^{-\Lambda H}\right)=1,
$$

where $\lambda=\Lambda / \sqrt{t}$ and $H=h / \sqrt{t}$. In the limit $H \rightarrow \infty$, we must recover the non-interacting regime; indeed, the limiting shape (27) reduces to (24).

In the third regime $h \ll \sqrt{t}$, the most appropriate description of the interface is in terms of $2 h$ random walkers. Adjacent walkers are separated by a large distance of the order of $\sqrt{t} / h$ and therefore are effectively non-interacting. We may compute the density of random walkers by solving the diffusion equation and the resulting limiting shape is given by the error function. This same prediction follows from the TDGL approach, as the factor $y_{x}^{2}$ can be neglected in the long-time limit (see Eq. (3)). Notice, however, that it is not possible to recover this limiting shape by taking the $H \rightarrow 0$ limit in Eq. (27). The reason for this non-analyticity is the large discrepancy between the horizontal and vertical scales when $\sqrt{t} \gg h$.

\section{B. Rectangular finger}

For the finger geometry, the minority phase initially occupies the semi-infinite region $y>0$ and $|x|<L$. The interesting regime is again $t \gg L^{2}$, where the two corners of the initial finger interact and the finger relaxes to a limiting shape that eventually recedes at constant velocity. In a reference frame moving with the finger, the interface $y(x)$ is thus stationary.

A new feature of the semi-infinite finger compared to the wedge geometry is the possibility for energy-lowering spin-flip events to occur. For example, if the tip of the finger contains a single spin, then when this spin flips, the fingertip irreversibly advances by one unit. Another new feature is that the finger can shed disconnected pieces whenever the tip of the finger has a width equal to one and length greater than two. Finally, the possibility of energy-lowering moves also means that the evolution of a finger is irreversible; there is no possibility of the system returning to its initial state once an energy-lowering move has occurred (Fig. 7).

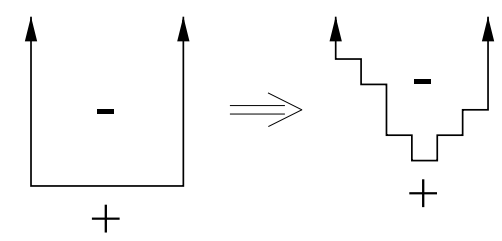

FIG. 7: Schematic evolution of a rectangular finger according to Ising-Glauber dynamics. On the right-hand side, the flip of the lowest minority spin is an irreversible process that causes the minimum height of the finger to advance by one.

Within the TDGL approach, $y(x)$ now satisfies the equation $D y_{x x}=v\left(1+y_{x}^{2}\right)$ [9, 20]. Integrating, and im- 
posing the boundary condition $y \rightarrow \infty$ when $|x| \rightarrow L$, we obtain, for the finger shape

$$
y=-\frac{2 L}{\pi} \ln \left[\cos \left(\frac{\pi x}{2 L}\right)\right]
$$

a result that was first apparently obtained by Mullins [9]. In this steady state, the finger recedes at a constant velocity that is given by $v=D y^{\prime \prime}(0)=\pi D / 2 L$.

Again, we test the applicability of the TDGL approach by comparing with numerical simulations. In our simulations of the finger, both energy conserving and energy lowering moves can occur, with rates 1 and 2, respectively. The rate at which the finger recedes is controlled by the fact that there is almost always an excess of two sites where the spin flip event $-\rightarrow+$ can occur (recession step) compared to $+\rightarrow-$. The only exception is the case where the fingertip width equals one; here the excess of potential recession steps over advancement steps also equals one. As a result of this nearly constant bias, the finger recedes at a constant rate with steady-state velocity equal to $1 / L$, up to exponentially small corrections.

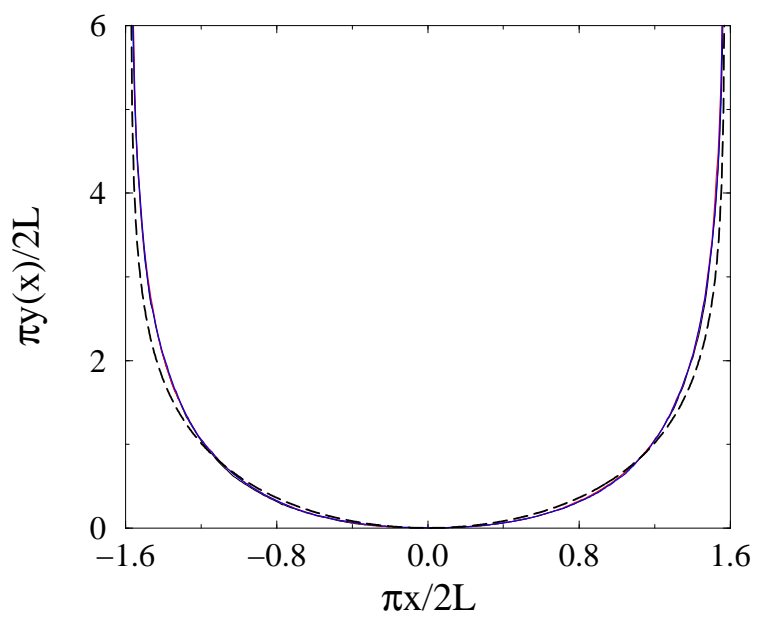

FIG. 8: Comparison of the finger shape predicted by the TDGL approach given in Eq. 30 (dashed line) with simulation results for 100 realizations of width $2 L=400$ at times $t=2 \times 10^{5}, 6 \times 10^{5}$, and $10^{6}$ steps (solid lines). The data for these three times essentially coincide.

While the shape of the finger is quantitatively close to the TDGL prediction, the discrepancy between the continuum theory and the simulations persists even as $L \rightarrow \infty$. This dichotomy is parallel to that observed in the wedge geometry.

We have not investigated the evolution of the interface subject to rules (ii) and (iii) in detail, so we just mention qualitative results. In both cases, the finger recedes at a constant velocity. For the system in a magnetic field (rule (iii)), $v(L)$ is a decreasing function of $L$ that saturates to a non-zero limit $v_{\infty}=\lim _{L \rightarrow \infty} v(L)>0$, in contrast to the case finger evolution in zero magnetic field. In the evaporation-dominated case, we estimate the velocity as the probability for the finger to reach the state where the fingertip has a width equal to one. In this case, an irreversible energy-lowering move can occur, so that the finger recedes by one step. If $1+\epsilon$ is the ratio of the evaporation to deposition rate, then $v(L) \sim e^{-\epsilon L}$.

\section{FLUCTUATIONS}

In addition to the mean interface shape, we also study fluctuations of the interface. Perhaps the simplest such quantity is the fluctuation in the area $S_{t}$ bounded by the interface at time $t$. We estimate the probability distribution of the area by the following simple argument. In the long-time limit, there are $N_{+} \sim t^{1 / 2}$ spins along the interface that can flip and join the majority phase and $N_{-}=N_{+}-1$ interface spins that can flip and join the minority. Thus the evolution of $S_{t}$ is driven by a deterministic contribution of rate one and a random contribution whose rate is of the order of $t^{1 / 4}$. This suggests that the evolution of $S_{t}$ is governed by the Langevin equation:

$$
\frac{d S_{t}}{d t}=1+t^{1 / 4} \xi(t)
$$

with $\xi(t)$ a random noise term that satisfies $\langle\xi(t)\rangle=0$ and $\left\langle\xi(t) \xi\left(t^{\prime}\right)\right\rangle=\delta\left(t-t^{\prime}\right)$. From this equation we immediately find that $\left\langle S_{t}\right\rangle=t$, while the fluctuation in $S_{t}$ is given by

$$
\begin{aligned}
\left\langle\left(S_{t}-t\right)^{2}\right\rangle & =\int_{0}^{t} d t_{1} \int_{0}^{t} d t_{2}\left(t_{1} t_{2}\right)^{1 / 4}\left\langle\xi\left(t_{1}\right) \xi\left(t_{2}\right)\right\rangle \\
& =\int_{0}^{t} d t_{1} t_{1}^{1 / 2} \propto t^{3 / 2} .
\end{aligned}
$$

Thus $S_{t}-t$ is a Gaussian random variable, and the probability distribution of the area $P_{n}(t) \equiv \operatorname{Prob}\left[S_{t}=n\right]$ is

$$
P_{n}(t) \propto \exp \left[-\frac{(n-t)^{2}}{t^{3 / 2}}\right] .
$$

The variance is proportional to $t^{3 / 4}$, in excellent agreement with our simulation results (Fig. 9). As a corollary to this latter result, the probability $P_{0}(t)$ for the interface to return to its original state decays as $P_{0}(t) \propto e^{-\sqrt{t}}$. Because $\int P_{0}\left(t^{\prime}\right) d t^{\prime}$ is finite, this means that the probability for the interface to eventually return to its original state is less than one 21].

We next consider fluctuations in the position of the interface by studying the location of the intersection of the interface with the diagonal $x=y$. The intersection between the diagonal and the (deterministic) limiting shape is $x=y=C_{1} \sqrt{t}$ with $C_{1}=(\sqrt{6} \ln 2) / \pi$. In each realization, however, $x$ is a random variable. Following the same argument as that applied for the total interface area, we anticipate that the variance in the position of the intersection point exhibits Gaussian fluctuations. This gives 


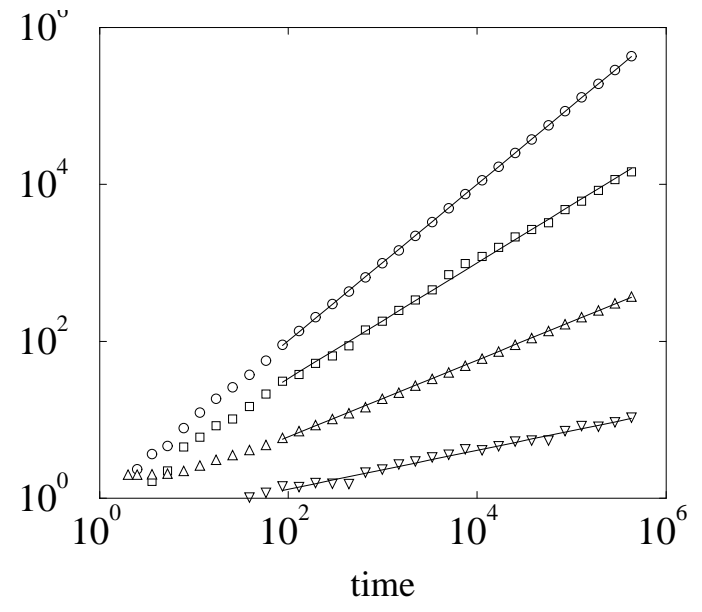

FIG. 9: Time-dependent properties of the Ising interface with Glauber kinetics for the wedge initial condition based on 100 realizations. Shown are: the average interface area $(0)$, the variance in this area $(\square)$, the mean value of the closest distance from the origin to the interface $(\Delta)$, and the variance in this distance $(\nabla)$. The thin solid lines are the best fits to the data at long time and have respective slopes 0.997, 0.737, 0.490 , and 0.248 .

$$
\left|x-C_{1} \sqrt{t}\right| \propto t^{1 / 4}
$$

again in excellent agreement with simulations (Fig. 9).

\section{DISCUSSION}

The dynamics of a single unbounded interface between ordered phases in the two-dimensional zero-temperature Ising-Glauber model has surprisingly rich properties and exciting connections with diverse topics in mathematics and physics. We discussed the correspondence to the limiting shapes of partitions 16, 22]; two other connections are to exclusion processes 23], and potentially to random matrices 24]. In particular, we presented evidence that in the case where the interface achieves an equilibrium state, the resulting interface coincides with the limiting shape in the partitioning problem.

The connection between the interface in the wedge geometry and exclusion processes arises from a simple construction in which one associates a particle with each vertical portion of the interface and a hole with each horizontal portion (Fig. 10). Thus the wedge geometry corresponds to an initial state in the particle system that consists of a semi-infinite line of particles in the region $(-\infty, 0)$ and empty space for $(0, \infty)$. Basic features of the interface shape can therefore be translated to corresponding properties of the particle density profile.

A possible connection to random matrices is best appreciated for the situation where the Ising interface grows irreversibly (our rule (i)). In this case, the weight of any

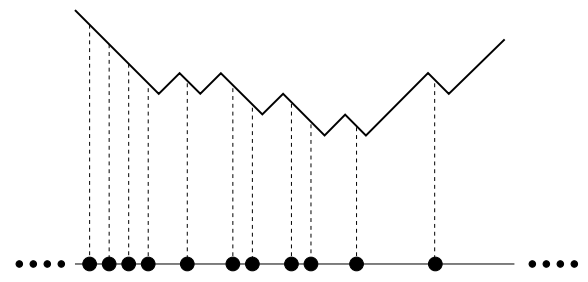

FIG. 10: The interface configuration of Fig. 3 rotated by $45^{\circ}$ and the corresponding particle configuration.

interface configuration is the number of ways to grow the final state from the empty diagram $\emptyset$ by adding squares one at a time, such that a partition is maintained at each step. For each partition $\pi=\left(y_{0} \geq y_{1} \geq \ldots \geq 0\right)$ of size $|\pi|=\sum y_{j}=N$, the number of distinct growth histories is usually denoted $\operatorname{dim} \pi$ [25] and irreversible interface growth corresponds to finding the limiting shape with the probability measure

$$
\operatorname{Prob}(\pi)=\frac{\operatorname{dim} \pi}{Z_{N}},
$$

where $Z_{N}=\sum_{|\mu|=N} \operatorname{dim} \mu[26]$. For the closely related Plancherel measure, $\operatorname{Prob}(\pi) \propto(\operatorname{dim} \pi)^{2}$, the limiting shape is also known 27, 28]. As explained in Ref. 22], the simplicity of the Plancherel measure stems from a hidden connection to unitary Gaussian random matrices; similarly, the measure (34) appears to be related to orthogonal Gaussian random matrices.

Another open question is the limiting shape of an initially large rectangle of down spins in an infinite sea of up spins. As mentioned in the introduction, although the time scale for this object to disappear is known [2], the shape of this object is not. One might expect that an initial square would evolve to a circular shape. It has indeed been proved that for curvature-driven growth every smooth closed curve in the plane asymptotically approaches a (shrinking) circular shape [11, 12]. The situation in three dimensions is much richer because a surface with both concave and convex portions can undergo fission by curvature-driven growth. The analogous result to Grayson's theorem is that any convex domain will ultimately approach a shrinking sphere [10]. On the other hand, for the Ising-Glauber model, nothing has been established rigorously. Our analytic and numerical results showed that lattice anisotropy effects persist in the interface dynamics. Thus we expect that a shrinking cluster in the long-time limit will not be isotropic.

Moving to three dimensions, it should be worthwhile to investigate the shape of the zero-temperature IsingGlauber interface on the cubic lattice when the spins in the positive octant $x \geq 0, y \geq 0, z \geq 0$ have a different sign than all other spins. We anticipate that the results will be similar to the limiting shape of the so-called plane partitions. In this latter problem, analytical results recently obtained in Refs. 29, 30] correspond to the case of the uniform measure. It would be interesting to study 
the possible correspondence between these plane partitions and the Ising interface.

Finally, we cannot resist mentioning that the finger shape (30) is mathematically identical to the SaffmanTaylor finger in the Hele-Shaw cell 31].

We are grateful to K. Kornev and R. Rajesh for helpful remarks and especially to B. Meerson for helpful comments on the manuscript and for bringing Ref. [9] to our attention. PLK and SR thank NSF grant DMR0227670 for financial support of this research. The research of JT was supported by a travel grant from ENS de Cachan.

\section{APPENDIX A: TRIANGULAR LATTICE}

We study the triangular lattice [32] because, in contrast to the square lattice, it is macroscopically isotropic, and hence one might anticipate that interface dynamics should be described by the intrinsically isotropic TDGL equation. We will see, however, that this is not the case.

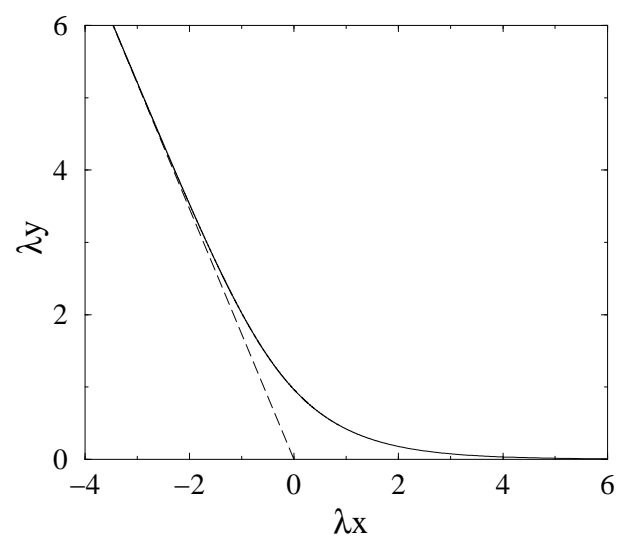

FIG. 11: The interface for the triangular lattice predicted by Eq. (A2) for the $2 \pi / 3$ wedge (dashed line).
There are two natural possibilities for a wedge geometry on the triangular lattice: opening angle (a) $\pi / 3$ and (b) $2 \pi / 3$. The latter case is more amenable to analysis, since energy decreasing spin flips can never occur and the system has the same reversibility as the $\pi / 2$ wedge on the square lattice.

Our derivation of the interface shape follows the steps given for the $\pi / 2$ wedge on the square lattice. The only new feature is that $a=\left(x_{2}-x_{1}\right)-\left(y_{1}-y_{2}\right) / \sqrt{3}$ and $b=2\left(y_{1}-y_{2}\right) / \sqrt{3}$ should be employed in Eq. (18). The variational problem on the triangular lattice involves maximizing the functional $G_{\lambda}[y]=\int_{0}^{\infty} d x L\left(y, y_{x}\right)$ with the associated Lagrangian

$$
\begin{aligned}
L\left(y, y_{x}\right) & =\frac{2}{\sqrt{3}} y_{x} \ln \frac{-2 y_{x}}{\sqrt{3}-y_{x}} \\
& -\left(1+\frac{1}{\sqrt{3}} y_{x}\right) \ln \frac{\sqrt{3}+y_{x}}{\sqrt{3}-y_{x}}-\lambda y .
\end{aligned}
$$

The Euler-Lagrange equation reduces to

$$
\frac{d}{d x}\left[\ln \frac{y_{x}^{2}}{3-y_{x}^{2}}\right]=-\sqrt{3} \lambda
$$

Integrating twice (subject to the boundary conditions $y \rightarrow 0$ for $x \rightarrow \infty$ and $y \rightarrow-\sqrt{3} x$ for $x \rightarrow-\infty$ ) yields

$$
x=-\frac{y}{\sqrt{3}}-\frac{2}{\sqrt{3} \lambda} \ln \left[1-e^{-\lambda y}\right], \quad \lambda=\frac{\pi}{3^{3 / 4} \sqrt{t}},
$$

where $\lambda$ is again determined from the constraint that the area between the limiting shape and the initial wedge equals $t$. To simplify this computation, it is useful to write the area in the form $\int_{0}^{\infty} d y\left(x+3^{-1 / 2} y\right)=t$.

The limiting shape of the interface is shown in Fig. 11] The asymptotic tails of the interface are again exponentially small. This agrees with simulations and contradicts to the Gaussian tails predicted by the TDGL approach.
[1] For reviews, see, e.g., J. D. Gunton, M. San Miguel, and P. S. Sahni in: Phase Transitions and Critical Phenomena, Vol. 8, eds. C. Domb and J. L. Lebowitz (Academic, NY 1983); A. J. Bray, Adv. Phys. 43, 357 (1994).

[2] I. M. Lifshitz, Sov. Phys. JETP 15, 939 (1962); L. Chayes, R. H. Schonmann, and G. Swindle, J. Stat. Phys. 79, 821 (1995).

[3] R. J. Glauber, J. Math. Phys. 4, 294 (1963).

[4] During the evolution, small bubbles can branch off the the domain, so strictly speaking there may be more than one interface.

[5] A very recent account of this and related fluid spreading problems appears in P. B. Warren, cond-mat/0311216

[6] According to zero-temperature Glauber dynamics, a patch of minority phase that occupies a domain $\mathcal{D}$ can grow up to the rectangular envelope of $\mathcal{D}$ (i.e., the smallest rectangle that contains $\mathcal{D}$ ). In the present case the rectangular envelope is always the original quadrant.

[7] S. M. Allen and J. W. Cahn, Acta Metall. 27, 1085 (1979).

[8] Note that the Allen-Cahn equation (11) is deterministic; generally, the TDGL equation is stochastic at positive temperature but becomes deterministic at zero temperature. Glauber dynamics remains stochastic even at $T=0$.

[9] W. W. Mullins, J. Appl. Phys. 27, 900 (1956).

[10] G. Huisken, J. Differential Geom. 20, 237 (1984).

[11] M. E. Gage and R. S. Hamilton, J. Differential Geom. 23, 69 (1986).

[12] M. A. Grayson, J. Differential Geom. 26, 285 (1987).

[13] D. L. Chopp and J. A. Sethian, Exper. Math. 2, 235 (1993); D. L. Chopp, Exper. Math. 3, 1 (1994).

[14] W. Fulton, Young Tableaux: With Applications to Representation Theory and Geometry (Cambridge University Press, New York, 1997). 
[15] G. E. Andrews, The Theory of Partitions (AddisonWesley, Reading, Mass., 1976).

[16] A. Vershik, Func. Anal. Appl. 30, no. 2, 90 (1996).

[17] Furthermore, $S_{t}$ is not a fixed number but a random variable that fluctuates from realization to realization; this apparently has an asymptotically negligible effect as the relative fluctuations in $S_{t}$ decay to zero (see Sect. V).

[18] S. Shlosman, J. Math. Phys. 41, 1364 (2000).

[19] R. Rajesh and D. Dhar, cond-mat/0303577

[20] A. Peleg, B. Meerson, A. Vilenkin, and M. Conti, Phys. Rev. E 63, 066101 (2001).

[21] S. Redner, A Guide to First-Passage Processes (Cambridge University Press, New York, 2001).

[22] A. Okounkov, math-ph/0309015

[23] See e.g., M. Praehofer and H. Spohn in "In and Out of Equilibrium", ed. V. Sidoravicius, Progress in Probability, 51, 185-204, (Birkhauser, Boston, 2002).

[24] M. L. Mehta, Random Matrices (Academic Press, Boston, 1991).
[25] The irreducible representations of the symmetric group $S_{N}$ are indexed by partitions $\pi$ of $\operatorname{size} N$, and $\operatorname{dim} \pi$ is the dimension of the corresponding representation [14].

[26] The normalization factor can be found from the generating function $\sum_{N \geq 0} \frac{Z_{N}}{N !} t^{N}=\exp \left(t+\frac{t^{2}}{2}\right)$.

[27] A. Vershik and S. Kerov, Sov. Math. Dokl. 18, 527 (1977).

[28] B. F. Logan and L. A. Shepp, Adv. Math. 26, 206 (1977).

[29] R. Cerf and R. Kenyon, Commun. Math. Phys. 222, 147 (2001).

[30] A. Okounkov and N. Reshetikhin, J. Amer. Math. Soc. 16, 581 (2003).

[31] P. G. Saffman and G. I. Taylor, Proc. R. Soc. London Ser. A 245, 312 (1958).

[32] The third possible two-dimensional lattice, the hexagonal, has uninteresting zero-temperature dynamics because the coordination number is odd. Thus any patch containing a hexagon never disappears. 